\title{
Amylase-Producing Maltooligosaccharide Provides Potential Relief in Rats with Loperamide-Induced Constipation
}

\author{
Eun Yeong Jang, ${ }^{1}$ Yejin Ahn, ${ }^{1}$ Hyung Joo Suh $\mathbb{D}^{,},{ }^{2}$ Ki-Bae Hong, ${ }^{3}$ and Kyungae Jo $\mathbb{D}^{3}$ \\ ${ }^{1}$ Department of Integrated Biomedical and Life Science, Graduate School, Korea University, Seoul 02841, Republic of Korea \\ ${ }^{2}$ Department of Public Health Science, Graduate School, Korea University, Seoul 02841, Republic of Korea \\ ${ }^{3}$ BK21 Plus, College of Health Science, Korea University, Seoul 02841, Republic of Korea \\ Correspondence should be addressed to Hyung Joo Suh; suh1960@korea.ac.kr and Kyungae Jo; kyungae11@korea.ac.kr
}

Received 25 May 2020; Revised 27 July 2020; Accepted 20 August 2020; Published 27 August 2020

Academic Editor: Min Li

Copyright (c) 2020 Eun Yeong Jang et al. This is an open access article distributed under the Creative Commons Attribution License, which permits unrestricted use, distribution, and reproduction in any medium, provided the original work is properly cited.

\begin{abstract}
Constipation is a chronic disease caused by infrequent, inadequate, and difficult bowel movements. The present study aimed to evaluate the potential laxative effect of maltooligosaccharide (MOS) on loperamide-induced constipation in a rat model. In vitro experiments were conducted to evaluate the effect of MOS on the growth of lactic acid bacteria. Moreover, to examine the effect of MOS administration on Sprague-Dawley (SD) rats with loperamide-induced constipation, the drinking water for the rats was supplemented with $10 \%$ or $15 \%$ of MOS for 14 days, and, thereafter, the improvement in constipation was assessed. For this, the rats were divided into five groups: normal (Nor), loperamide-induced constipated (Con), positive control (15\% of dual-oligosaccharide (DuO-15)), 10\% MOS treated (MOS-10), and 15\% MOS-treated (MOS-15). In an in vitro test, MOS treatment promoted the growth of lactic acid bacteria except Lactobacillus bulgaricus. Treatment with higher MOS dose relieved constipation in rats by improving the fecal pellet and water content. Furthermore, in the high MOS dose group, the cecal short-chain fatty acid levels significantly increased compared to those in the control group $(P<0.001)$. MOS treatment also improved the mucosal thickness as well as mucin secretion and increased the area of intestinal Cajal cells compared to that in the control group $(P<0.001)$. These findings suggest that MOS relieves constipation and has beneficial effect on the gastrointestinal tract, and, therefore, it can be used as an ingredient in functional foods for treating constipation or improving intestinal health.
\end{abstract}

\section{Introduction}

As people have become more concerned about their health, consumption of healthier foods has increased, thereby reducing disease risk. In this sense, certain types of dietary carbohydrates, particularly functional oligosaccharides, have gained immense interest. Oligosaccharides are low-molecular carbohydrates comprising 3-10 monosaccharides, such as glucose, fructose, and galactose [1]. Functional oligosaccharides such as galactooligosaccharide (GOS), fructooligosaccharide (FOS), and maltooligosaccharide (MOS) are well-known prebiotics owing to their ability to selectively stimulate beneficial bacteria in the intestines, particularly bifidobacterial species $[2,3]$. These oligosaccharides increase the production of short-chain fatty acids by bifidobacteria and further stimulate intestinal peristalsis and increase fecal water content through osmotic pressure [4]. The most abundant sources of functional oligosaccharides are seaweeds and plants.

Starch, a carbohydrate stored in higher plants, is a homopolysaccharide comprising two types of glucose polymers, amylose and amylopectin. Amylose is an unbranched homopolysaccharide with $\alpha$ - $(1 \longrightarrow 4)$ glycosidic linkages, whereas amylopectin is a branched homopolysaccharide with $\alpha$ - $(1 \longrightarrow 4)$ and $\alpha$ - $(1 \longrightarrow 6)$ glycosidic linkages. Starch acts as a substrate for producing oligosaccharides. MOS, produced by $\alpha$-amylase (EC 3.2.1.1) and pullulanase (EC 3.2.1.9), is a mixture of linear oligosaccharides comprising two (G2), three (G3), four (G4), five (G5), or six (G6) glucose units joined by $\alpha$-(1 $\longrightarrow 4)$ 
glycosidic linkages [5]. MOSs serve as digestive nutritional sweeteners with low calorie content and osmotic pressure and are widely used in processing functional foods [6]. They display physiological characteristics that relieve fatigue, improve visceral peristalsis, and prevent constipation [3].

The MOS used in this study was prepared using only $\alpha$-amylase without pullulanase. MOS containing undigested amylopectin has prebiotic activity that selectively stimulates the growth and function of the intestinal microflora. This type of alpha-amylase-resistant starch has prebiotic properties similar to those of resistant starch. Microflora changes resulting from the MOS-supplemented diet are beneficial to the digestive tract. Previous studies on constipation focused on fiber and nondigested oligosaccharides $[7,8]$. In particular, isomaltooligosaccharide (IMOS) produced via hydrolysis and transglycosylation from soluble starch has been used to treat chronic constipation, and Goulas et al. [9] reported that at least $8 \mathrm{~g} /$ day of IMOS would be required to substantially elevate the bifidobacterial cell number in the human gut. Moreover, administration of $90 \%$ IMOS at a concentration of $8 \mathrm{~g} / \mathrm{day} / \mathrm{kg}$ of body weight in mice with loperamide-induced constipation increased the water content of the stool, shortened intestinal transit time, and increased stool short-chain fatty acid (SCFA) concentration [10]. Similar to MOS, IMOS is prepared using starch as the raw material; however, the process is complex as it requires a combination of enzymes immobilized in a two-stage reactor. Previous studies demonstrated that the laxative effect of MOS was similar to IMOS; however, the difference was not significant. Additionally, no further studies confirmed the effect of MOS is low doses. Therefore, in the present study, we evaluated the laxative potential of MOS with $\alpha$-amylaseresistant starch prepared from organic rice.

\section{Materials and Methods}

2.1. Experimental Animals and Reagents. The Institutional Animal Care and Use Committee of Korea University (KUIACUC; Approval Number KU-2019-0012) approved the use of animals for this study. The animals used in the experiment were Sprague-Dawley (SD) male rats (weighing $160-180 \mathrm{~g}$ ) at 6 weeks of age (OrientBio; Seongnam, Korea). After adapting to the environment for 7 days, the experimental animals were randomly divided into 5 groups of 8 rats each, and each rat was bred for 25 days in an individual cage. During the experimental period, water and food were provided ad libitum at a room temperature of $20-22^{\circ} \mathrm{C}$ and a relative humidity of $50 \%-55 \%$ under a $12 \mathrm{~h}$ light/dark cycle. The MOS and dual-oligosaccharide ( $\mathrm{DuO})$ were supplied by NEO CREMAR Co. Ltd. (Seoul, Republic of Korea). Loperamide (L4762) was purchased from Sigma-Aldrich (St. Louis, MO, USA).

2.2. Bacterial Strains and Growth Conditions. Lactobacillus fermentum KCTC 3112, L. paracasei KCTC 3510, L. reuteri KCTC 3594, L. bulgaricus KCTC 3536, Bifidobacterium breve KCTC 3220, and B. lactis KCTC 5854 were purchased from the Korean Collection for Type
Cultures (Daejeon, Republic of Korea) for evaluating the effect of MOS on the proliferation of lactic acid bacteria. Lactic acid bacteria were inoculated into modified peptone yeast extract fructose medium (PYF) containing $1 \%, 2 \%$, or $4 \%$ of MOS and incubated at $37^{\circ} \mathrm{C}$ for $48 \mathrm{~h}$. To determine the bacterial growth rate, culture samples were collected at $12 \mathrm{~h}$ intervals and absorbance was measured at $660 \mathrm{~nm}$.

\subsection{Determination of MOS Constipation Mitigation Effect.} After one week of adaptation, constipation was induced by intraperitoneal administration of loperamide $(3 \mathrm{mg} / \mathrm{kg})$ once a day for 6 days in the experimental groups except for the untreated control group (Nor). The mice with constipation were divided into negative control (Con), positive control (DuO-15), low-dose MOS administration (MOS-10), and high-dose MOS administration (MOS-15) groups. The Nor and Con groups were supplied with only drinking water during the 2-week recovery period, whereas the $\mathrm{DuO}$, MOS10 , and MOS-15 groups were supplied with drinking water supplemented with $15 \%$ of $\mathrm{DuO}, 10 \%$ of MOS, and $15 \%$ of MOS, respectively. Rats were sacrificed using carbon dioxide $\left(\mathrm{CO}_{2}\right)$, and intestinal and cecum were collected for further analysis.

2.4. Measurement of Fecal Parameters. Fecal samples were collected thrice a week at 10 am during the sample processing period. The number of fecal pellets and wet fecal weight were measured. Fecal water content was calculated by drying the stool in an oven at $60^{\circ} \mathrm{C}$ for $24 \mathrm{~h}$, measuring the dry weight, and calculating the difference between the wet and dry fecal weights.

2.5. Intestinal Transit Ratio. Intestinal transit ratio of MOS was measured using a modified method based on that of Kim et al. [11]. To investigate the effect of MOS on dietary transport, after 2 weeks of sample treatment, $1 \mathrm{ml}$ of $8 \%$ (W/V) activated carbon was orally administered to the rats, and after $30 \mathrm{~min}$, the gastrointestinal tract was extracted. The length of the intestine was calculated by making an incision after measuring the lengths of the small and large intestine. The intestinal transit ratio was calculated by dividing the total intestinal distance by the distance traveled by activated charcoal.

2.6. Determination of SCFAs in Feces. To determine the SCFA content, $1 \mathrm{~g}$ of cecal contents was collected 1 day before the end of the experiment, followed by SCFA extraction using $5 \mathrm{ml}$ of methanol and filtering via a $0.45 \mu \mathrm{m}$ Millipore filter (Millipore, USA). SCFAs were analyzed using a gas chromatography (GC) system (Agilent Technologies, Santa Clara, CA, USA) equipped with a GC column (DBFFAP $123-3253,50 \mathrm{~m} \times 0.32 \mathrm{~mm} \times 0.50 \mu \mathrm{M}$ ), flame ionization detector, and autosampler. Nitrogen was used as the carrier gas with a flow rate of $1.4 \mathrm{ml} / \mathrm{min}$ a split ratio of $10: 1$. The sample injection volume was $1 \mu \mathrm{l}$, and inlet and detector temperatures were $200^{\circ} \mathrm{C}$ and $240^{\circ} \mathrm{C}$, respectively, based on the protocol by Demingne and Remesy [12]. Acetic acid, 
propionic acid, and butyric acid contents were used as standards.

2.7. Histopathological Analysis. Colon tissue obtained from the sacrificed SD rats was fixed with $10 \%$ formalin for $24 \mathrm{~h}$. The colon was embedded in paraffin wax and then sectioned into $3 \mathrm{~mm}$ thick slices. The slices were stained with hematoxylin and eosin (H\&E; Sigma-Aldrich Co, St. Louis, MO, USA). Morphological features of intestinal mucosa cells were observed via light microscopy (ZEISS, Axiovert S100, Germany).

For mucin staining, tissue sections fixed in paraffin $(3 \mu \mathrm{m})$ were deparaffinized with xylene. After deparaffinization, the tissues were washed with distilled water and stained with Alcian blue for $30 \mathrm{~min}$. Eventually, the morphology of the cryptic cells in the stained colon sections was observed via light microscopy (ZEISS, Axiovert S100, Germany).

2.8. Immunohistochemistry Examination. Interstitial cells of Cajal (ICC) are cells distributed in the smooth muscle layer of the intestine that assists in intestinal peristalsis [13]. To observe these cells, paraffin-embedded intestinal tissues were cut into $4 \mu \mathrm{m}$ sections after staining with $\mathrm{H} \& \mathrm{E}$. The sections were then deparaffinized using xylene and hydrolyzed for 5 min with different concentrations of ethanol (100\%, 90\%, $80 \%$, and $70 \%$ ). Slide glass containing the tissue was heated to $98^{\circ} \mathrm{C}$ for $20 \mathrm{~min}$ for antigen retrieval, followed by preantibody blocking to prevent staining of cells other than ICC. Primary antibody was diluted 1:200 using AB c-kit (Santa Cruz; SC168 , Dallas, TX, USA) and reacted at $4^{\circ} \mathrm{C}$ for 1 day. After the reaction, antibody enhancer was added to the tissue and incubated for $10 \mathrm{~min}$. After $5 \mathrm{~min}$ of treatment with chromogen (DAB Substrate $1 \mathrm{ml}+\mathrm{DAB}$ chromogen 1-2 drops), the sections were washed in running water for 1-2 min, reacted with hematoxylin, and then washed with PBS and running water, and eventually they were mounted. Stained ICCs were observed under an optical microscope (ZEISS, Axiovert S100, Jena, Germany), and the number of pixels with RGB values was determined using MATLAB.

2.9. Statistical Analysis. Data was statistically analyzed using the statistical package for social science (version 12.0). Means and standard deviations were calculated for all measurements. Following one-way analysis of variance (ANOVA), the significance of intergroup differences was confirmed by Tukey's multiple range test at $P<0.05$.

\section{Results}

3.1. Utilization of MOS by Lactic Acid Bacteria In Vitro. MOS provided by NEO CREMAR comprised $48.8 \%$ of dietary fiber (data not shown). In total, $99 \%$ of the total dietary fiber was water-soluble. Polysaccharides are often fermented by intestinal microbes that may selectively use them. Nonprebiotic MOSs reveal different levels of polymerization, and, consequently, the availability of MOS in intestinal microbes may vary. Therefore, the availability of the used MOS for enteric microbes was measured in vitro (Figure 1). Figure 1 illustrates the growth of enteric bacteria in medium containing $0 \%-2 \%$ MOS. Lactic acid bacteria except for L. bulgaricus showed increased growth in correlation with the amount of MOS added. L. fermentum and Bifidobacterium lactis started multiplying rapidly $12 \mathrm{~h}$ after inoculation into MOS-supplemented medium, whereas L. paracasei and L. reuteri displayed a rapid increase in cell numbers for $12 \mathrm{~h}$ followed by a gradual decrease of the proliferation rate. The growth rate of $B$. breve increased $24 \mathrm{~h}$ after treatment commencement, and, in case of L. bulgaricus, MOS had little effect on the cell growth (Figure 1).

3.2. Fecal Pellet Number and Water Content. To evaluate the constipation mitigation effect of MOS, changes in the number of fecal pellets and fecal water content were measured. The number of fecal pellets was 39/day $(n=8)$ in the negative control group (Con), which was significantly lower than that in the normal group (48/day, $n=8)$, as illustrated in Figure 2(a) $(P<0.01)$. The positive control group (DuO-15), as well as the low and high MOS treated groups (MOS-10 and MOS-15) had lower numbers of fecal pellets than those in the normal group, but, within the three groups, the numbers of fecal pellets were similar $(42,44$, and 42 /day, $n=8$; Figure $2(\mathrm{a}))$.

The water content of fecal pellets was the lowest in the negative control group at $6.79 \%$, whereas in the positive control group it was $8.64 \%$, which was similar to $9.26 \%$ in the normal group. The fecal water contents of the groups treated with low and high concentrations of MOS $(12.74 \%$ and $15.34 \%$ ) were significantly higher than those in the normal group and increased in a concentration-dependent manner $(P<0.001$; Figure 2(b)).

3.3. Intestinal Transit Ratio. Intestinal transit ratio is an import indicator of constipation [14]. This ratio was measured to compare the effect of MOS on intestinal motility (Figure 3 ). In the constipated control group without MOS treatment, the intestinal ratio was $42.51 \%(n=8)$. Groups subjected to MOS treatment and the positive control group treated with dual-oligosaccharide presented an increased intestinal transit ratio compared to that in the control group. In particular, the group treated with a higher dose of MOS had a transit ratio of $53.92 \%$, which was substantially higher than that of the control group.

3.4. SCFA Changes in Cecum. SCFAs, such as acetic acid, propionic acid, and butyric acid, are rapidly absorbed by the intestinal mucosa and are used as a major energy source by colonic mucosa [15]. Therefore, to investigate the effect of MOS administration on the production of SCFAs that help promote intestinal health, acetic acid, propionic acid, butyric acid, and total SCFA contents in cecum were analyzed via GC (Figure 4). A high level of SCFA content was found in both normal and high dose MOS groups, with levels significantly different from the control group $(P<0.05$ and 


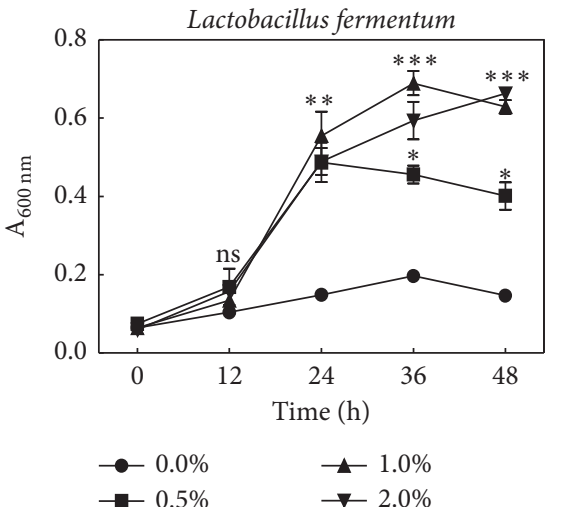

(a)

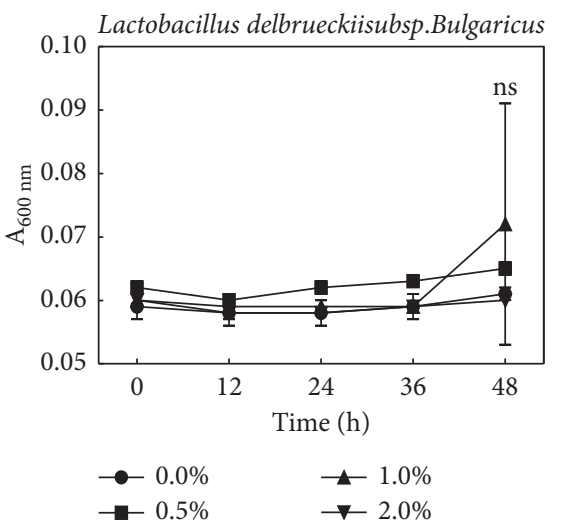

(d)

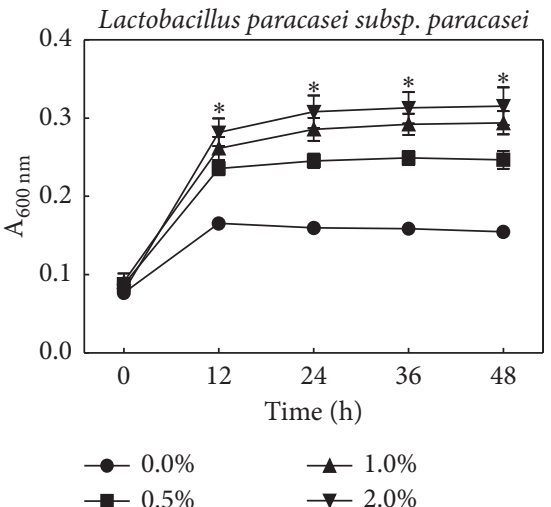

(b)

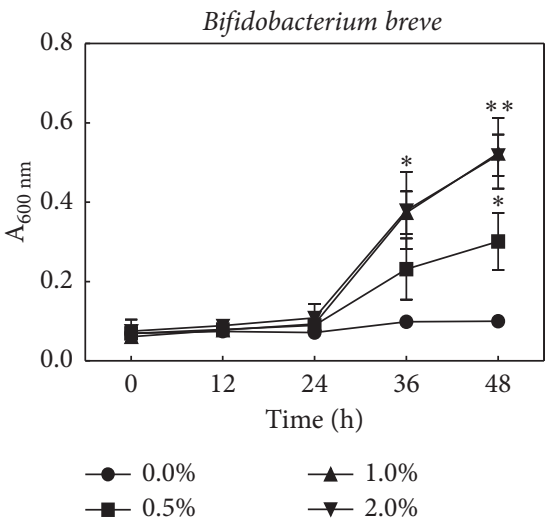

(e)

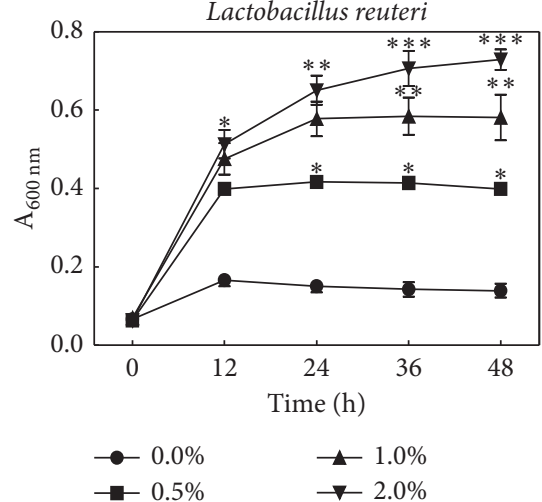

(c)

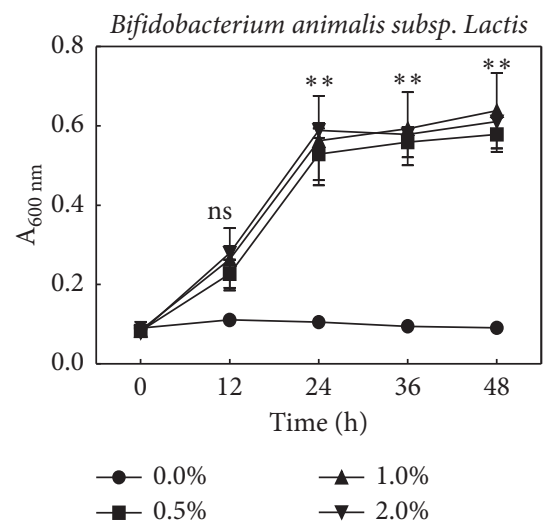

(f)

FIGURE 1: Growth of Lactobacillus fermentum, Lactobacillus paracasei, Lactobacillus reuteri, Lactobacillus bulgaricus, Bifidobacterium breve, and Bifidobacterium lactis in modified PYF broth containing various concentrations of MOS. Bars represent the standard deviation from triplicate determinations. ${ }^{*} P<0.05,{ }^{* *} P<0.01$, and ${ }^{* * *} P<0.001$ versus control group (ANOVA followed by post hoc Tukey's test). n.s.: not significant.

$P<0.001$, respectively). Among acetic acid, propionic acid, and butyric acid, the amount of acetic acid produced was the highest in all groups. SCFA production increased in correlation to the MOS dose. In the Duo-15 positive control group, an increase in SCFA content was detected in comparison to the control group.

\subsection{Changes in Histological Structure and Mucin Secretion of} the Colon. Figure 5 depicts the effect of MOS intake on mucosal layer thickness in rats with loperamide-induced constipation. The thickness of intestinal mucosa was the lowest in the constipated control group without MOS treatment, which also displayed the lowest intestinal transit ratio among the experimental groups. Both MOS treated groups and the positive control group revealed a higher mucosal layer thickness than that observed in the control group $(P<0.001)$, indicating that intestinal mobility was restored by MOS treatment. In conclusion, mucosal thickness tended to increase in positive correlation with MOS intake.

Alcian blue is used to stain crypt cells, which are involved in mucin production. As illustrated in Figure 6, the control group treated with loperamide alone presented a reduced level of Alcian blue stained area (presenting mucin) in the mucosal layer of the colon compared to that in the normal group; however, oligosaccharide (Duo-15, MOS-10, and MOS-15) uptake significantly increased the percentage of crypt cells involved in mucin production compared to that in the control group $(P<0.001)$.

3.6. Changes in the Area of ICC. ICCs regulate the smooth muscles in the intestinal mucosa and play an important role in controlling intestinal motility, including contraction and relaxation of the intestinal muscles [16]. ICC is present in all layers of the colon [17]. In the normal group, the c-kit positive immune response structure is presented in brown (Figure 7).

The constipated group without MOS treatment revealed a significant decrease in the ICC area compared to that in the normal group $(P<0.001$; Figure 7$)$. Both low dose of MOS and dual-oligosaccharide treatment significantly increased the ICC area in SD rats compared to that in the control rats $(P<0.01)$, and the ICC region significantly increased to the highest level, particularly when the high dose of MOS was administered $(P<0.001)$. 


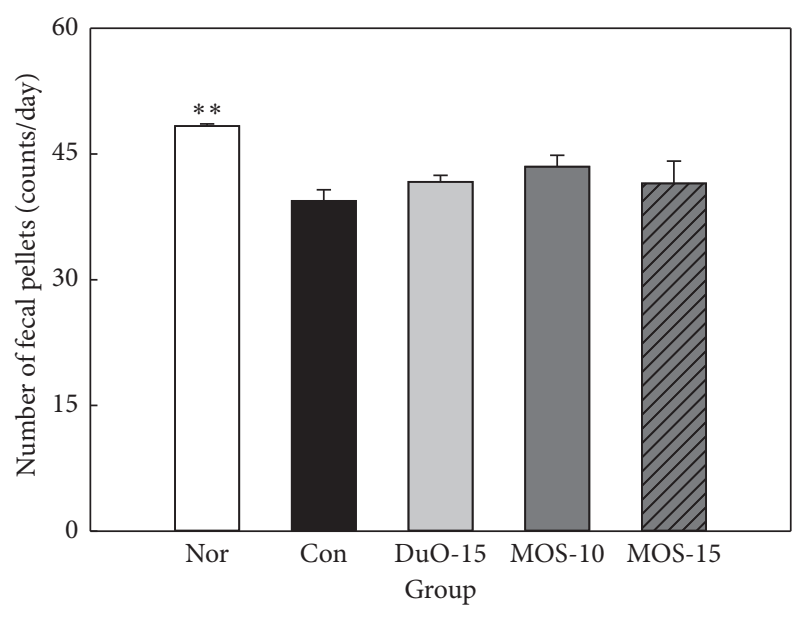

(a)

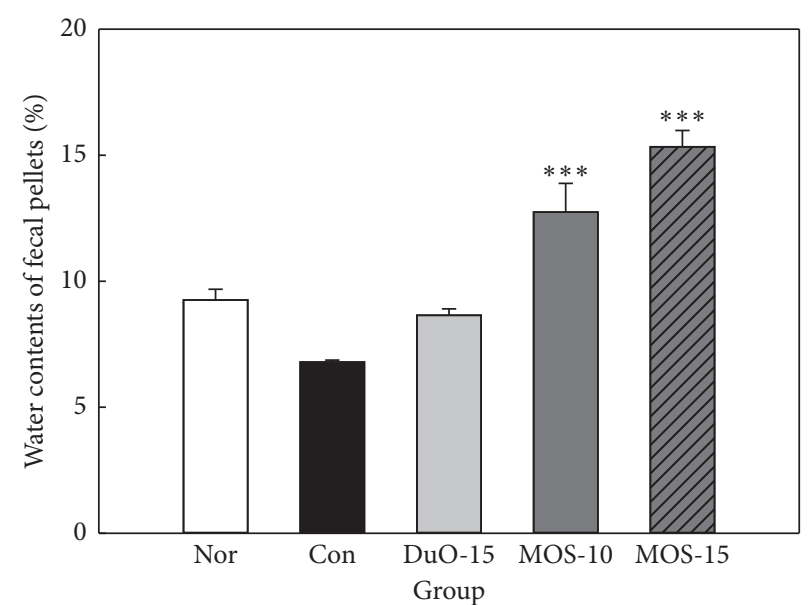

(b)

Figure 2: Effect of maltooligosaccharide (MOS) on fecal pellets number (a) and fecal water content (b) in normal and loperamideinduced constipated rats. Nor: normal group, Con: control group, DuO-15: Du-oligo 15\%-treated group as positive control, MOS-10: MOS 10\%-treated group, and MOS-15: MOS 15\%-treated group. The other groups except Nor are loperamide-induced constipation models. Data are represented as the mean \pm SE. ${ }^{* *} P<0.01$ and ${ }^{* * *} P<0.001$ versus control group (ANOVA followed by post hoc Tukey's test).

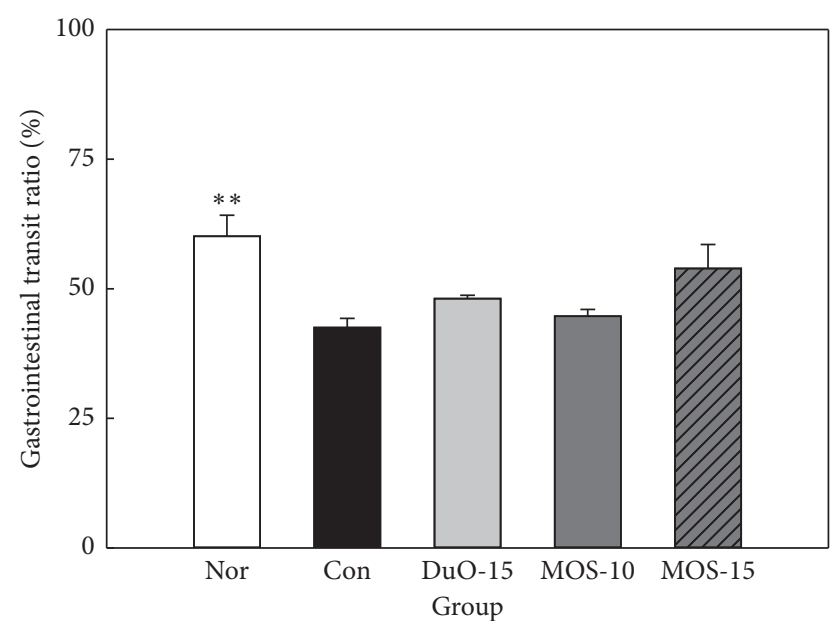

FIGURE 3: Effect of maltooligosaccharide (MOS) on gastrointestinal transit ratio in normal and loperamide-induced constipated rats. Nor: normal group, Con: control group, DuO-15: Du-oligo 15\%-treated group as positive control, MOS-10: MOS 10\%treated group, and MOS-15: MOS 15\%-treated group. The other groups except Nor are loperamide-induced constipation models. Data are represented as the mean $\pm \mathrm{SE} .{ }^{* *} P<0.01$ versus control group (ANOVA followed by post hoc Tukey's test).

\section{Discussion}

Constipation is a chronic disorder characterized by reduced bowel movements, difficulty in defecation, and incomplete intestinal evacuation sensation $[18,19]$. Constipation is often caused by a lack of dietary fiber, insufficient fluid intake, decreased physical activity, drug side effects, hyperthyroidism, and obstruction by colorectal cancer [20]. Altered diet leads to changes in the intestinal microflora, making it safe and sustainable to prevent or relieve constipation. Direct intake of probiotics can induce favorable

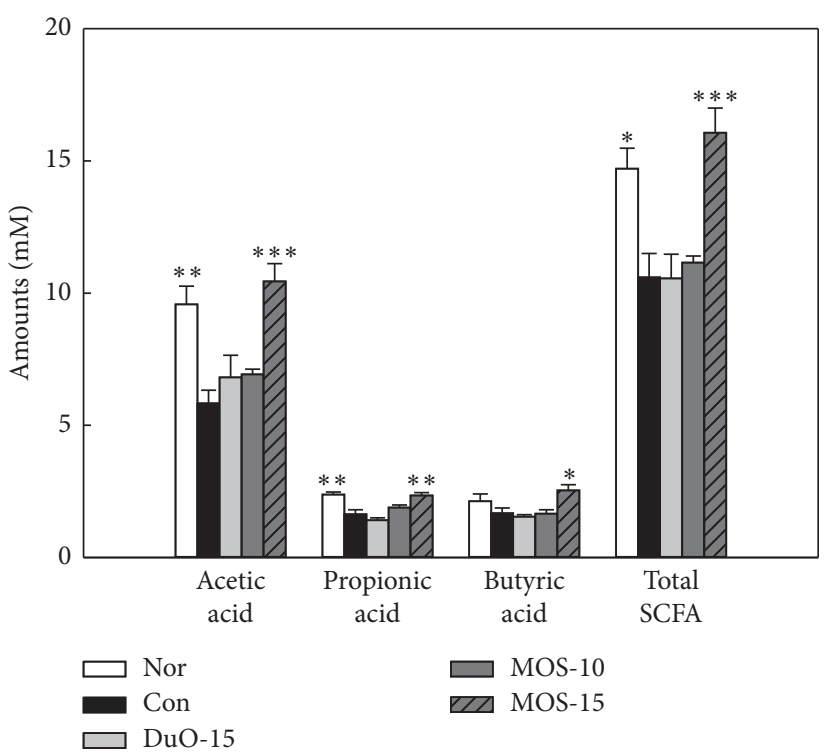

FIGURE 4: The concentration of acetic acid, butyric acid, propionic acid, and total short-chain fatty acid on cecum in normal and loperamide-induced constipated rats. Nor: normal group, Con: control group, DuO-15: Du-oligo 15\%-treated group as positive control, MOS-10: maltooligosaccharide 10\%-treated group, and MOS-15: MOS 15\%-treated group. The other groups except Nor are loperamide-induced constipation models. Data are represented as the mean $\pm \mathrm{SE} .{ }^{*} P<0.05,{ }^{* *} P<0.01$, and ${ }^{* * *} P<0.001$ versus control group (ANOVA followed by post hoc Tukey's test).

changes in the intestinal microflora and promote the growth of beneficial bacteria in the body. Another way to improve gut flora is the regular intake of prebiotics such as xylooligosaccharides, GOSs, and FOSs, which are used as nutrients by the gut microbes. Prebiotics improve the intestinal environment by promoting the growth of beneficial bacteria and inhibiting the growth of pathogenic bacteria. MOSs are 


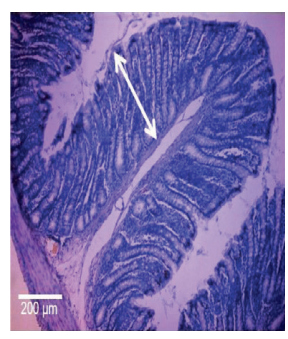

Nor

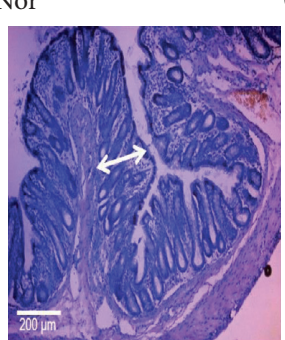

MOS-10

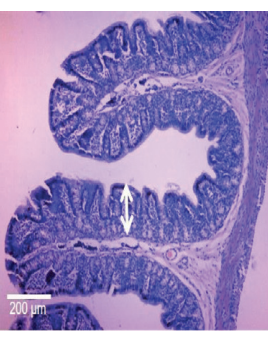

Con

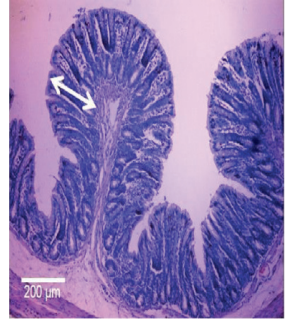

$\mathrm{DuO}-15$

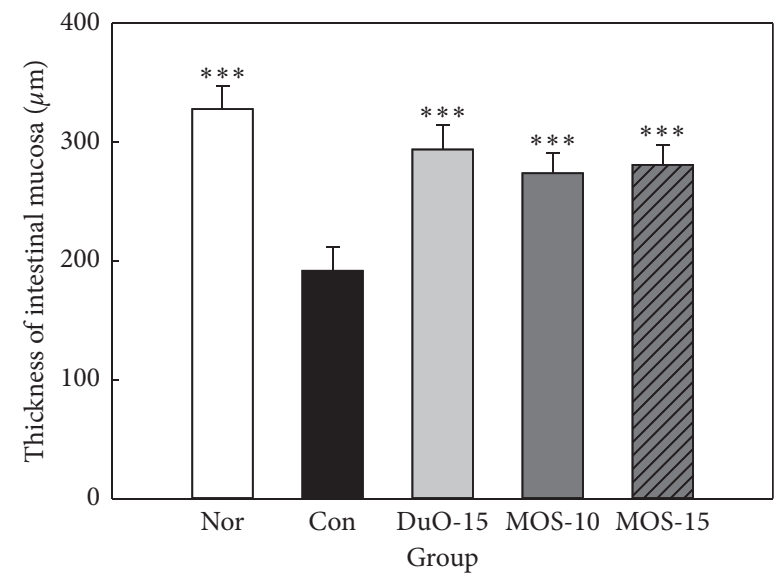

MOS-15

Figure 5: Effect of maltooligosaccharide (MOS) on thickness of intestinal mucosa. Nor: normal group, Con: control group, DuO-15: Duoligo 15\%-treated group as positive control, MOS-10: MOS 10\%-treated group, and MOS-15: MOS 15\%-treated group. The other groups except Nor are loperamide-induced constipation models. Data are represented as the mean \pm SE. ${ }^{* * *} P<0.001$ versus control group (ANOVA followed by post hoc Tukey's test).

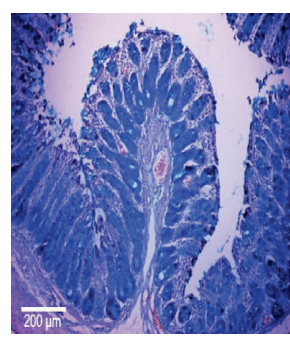

Nor

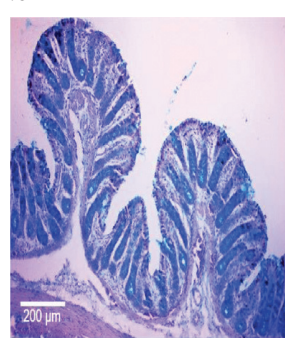

MOS-10

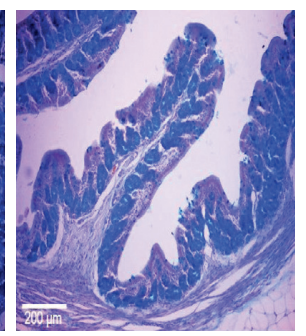

Con

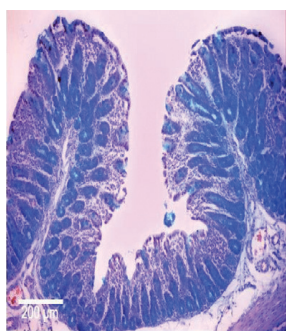

DuO-15

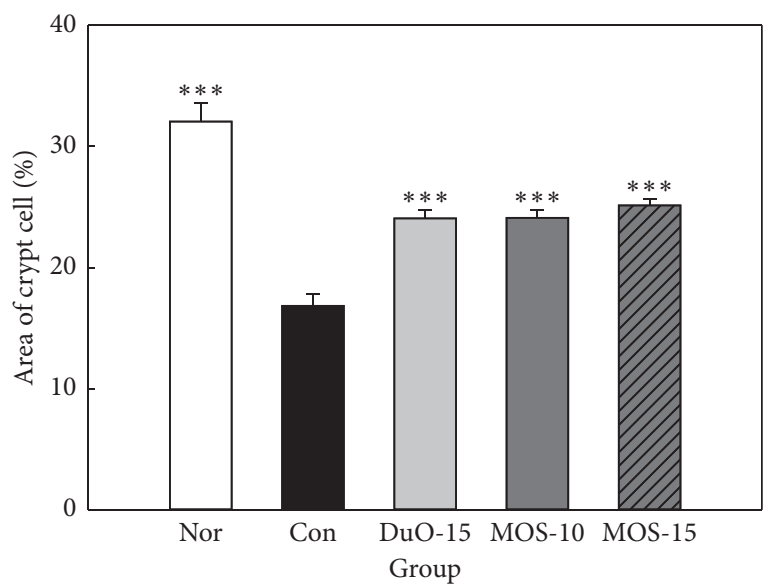

FIGURE 6: Effect of maltooligosaccharide (MOS) on area of crypt cells. Nor: normal group, Con: control group, DuO-15: Du-oligo 15\%treated group as positive control, MOS-10: MOS 10\%-treated group, and MOS-15: MOS 15\%-treated group. The other groups except Nor are loperamide-induced constipation models. Data are represented as the mean \pm SE. ${ }^{* * *} P<0.001$ versus control group (ANOVA followed by post hoc Tukey's test).

oligosaccharides produced during the hydrolysis of amylose, a major component in plant starch. In addition to amylase, pullulanase with amylopectin-degrading activity is used for MOS production; however, only amylase was used to manufacture the MOS used in the present study. The typical end products of $\alpha$-amylase activity are branched $\alpha$-limit dextrin and MOSs comprising 2-12 glucose units [21].

Changes in fecal pellet number, weight, and water content in rats with loperamide-induced constipation are important factors that need to be improved while aiming to relieve constipation. Previous studies have reported a marked reduction in the fecal pellet count, weight, and water content, in rats treated with loperamide [22, 23], whereas prebiotic intake is known to improve the fecal parameters [24, 25]. The MOS used in this study also improved the fecal pellet number and water content (Figures 2 and 3 ).

Compared to the constipated control group, high concentration MOS treatment tended to increase the gastrointestinal transit ratio (Figure 3). The effect of MOS on the 


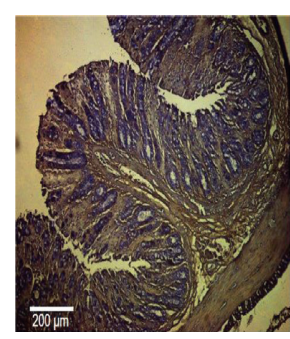

Nor

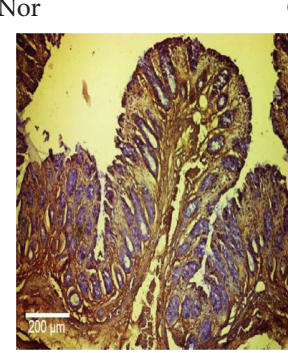

MOS-10

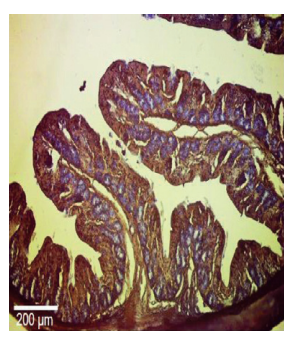

Con

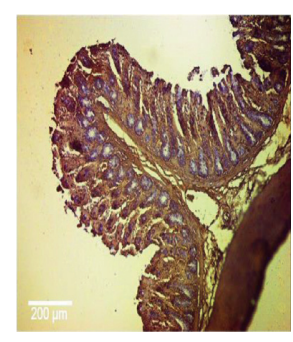

DuO-15

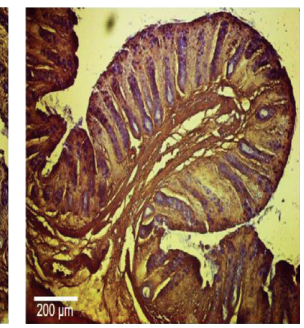

MOS-15

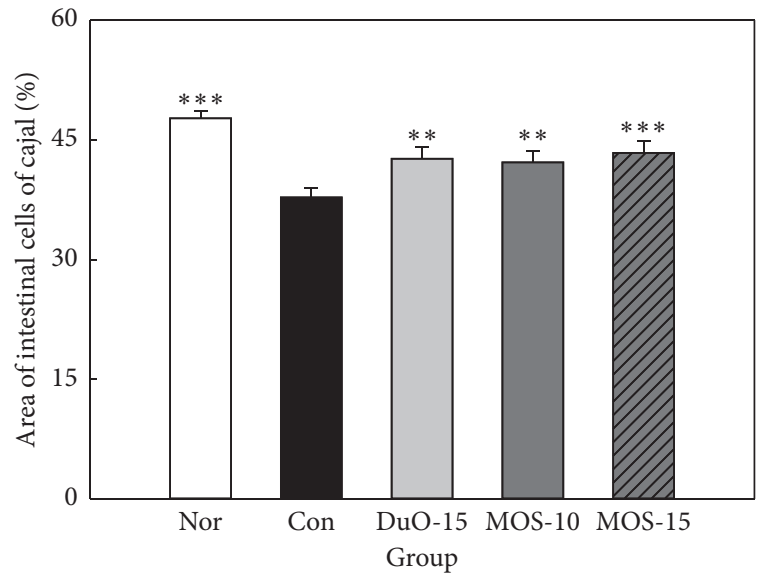

FIGURE 7: Effect of maltooligosaccharide (MOS) on the area of interstitial cells of cajal (ICC). Nor: normal group, Con: control group, DuO15: Du-oligo 15\%-treated group as positive control, MOS-10: MOS 10\%-treated group, and MOS-15: MOS 15\%-treated group. The other groups except Nor are loperamide-induced constipation models. Data are represented as the mean \pm SE. ${ }^{* *} P<0.01$ and ${ }^{* * *} P<0.001$ versus control group (ANOVA followed by post hoc Tukey's test).

intestinal transit ratio is presumably due to the limit dextrin. $\alpha$-amylase produces a mixture of limit $\alpha$-dextrins, short linear oligosaccharides, and glucose during MOS production [26]. Recently, nondigestible dextrin, $\alpha$-cyclodextrin, and dextran were observed to increase intestinal SCFA production, in particular, acetate and propionate, in an in vitro fecal fermentation model of human colonic microbiota [27]. Moreover, functional oligosaccharides can inhibit and alleviate intestinal diseases. For example, FOS, MOS, and GOS are known to be suitable substrates for Bifidobacterium sp. and Bacteroides sp. [28]. The production of SCFAs by these intestinal microorganisms stimulates intestinal peristalsis and increases the humidity of the stool by osmotic pressure [4]. In this study, MOS administration significantly increased the total SCFA content compared to that in the control $(P<0.001$; Figure 4$)$. Mammalian intestine has a complex microbial ecosystem that is not completely understood. Short-term [29] and long-term [30] dietary carbohydrate intake affects the human fecal microflora. The use of specific starches (e.g., complex resistant starch granules, soluble MOSs, and amylopectin) by intestinal microorganisms depends on the specific activity of the GH13 enzyme and the type of glycan absorption system that works with the enzyme [31].

In Figure 6, goblet cells are stained with Alcian blue in normal rats, and the fine cells in the crypts are wellaligned. This indicates that the goblet cells produce normal mucins containing sulfomucin. Loperamide administration significantly reduced the number of cryptic cells compared to those in the normal group $(P<0.001)$, indicating that it contained less mucin. Higher counts of unstained and stained goblet cells were observed in MOS-supplemented rats compared to those in the loperamide control group, indicating an increase in the goblet cells and intracellular mucin levels resulting in improved mucus secretion [32]. Mucin, a major component of lumen mucus, serves to protect the colonic mucosa from mechanical and chemical damage [33]. Loperamide reduces both synthesis and storage of mucins in cryptic cells [34]. As illustrated in Figure 6, MOS administration improved mucin production, thereby facilitating the passage of feces along the colon and reducing the risk of mucosal damage by decreasing the exposure time of colonic mucosa to potential risk factors.

Compared to the constipated control group without MOS treatment, the ICC count in the MOS treated groups was significantly higher (Figure $7, P<0.01$ and $P<0.001$, respectively). ICC plays a pivotal role in controlling intestinal motility and is present in all layers of the colon $[15,35]$. A decrease in ICC leads to slow bowel movements and decreased smooth muscle contractile activity, which can cause constipation. In addition, ICC numbers are markedly reduced during constipation, which can further amplify the constipation $[17,36]$.

\section{Conclusions}

In this study, MOS increased the water content of the stool, intestinal passage rate, and production of SCFAs, such as acetic acid, propionic acid, and butyric acid, in the intestinal tract. Moreover, it promoted mucus production by the epithelial cells of the small intestine and increased the number of ICC compared to that in the control rats without MOS treatment. MOS alleviated the symptoms of loperamide-induced constipation as it contributed to the stabilization of the intestinal mucosal barrier. Ingestion of MOS can be recommended as an effective alternative for treating constipation. 


\section{Data Availability}

The data used to support the findings of this study are included in the article.

\section{Conflicts of Interest}

The authors declare that there are no conflicts of interest regarding the publication of this paper.

\section{Authors' Contributions}

Eun Yeong Jang and Yejin Ahn contributed equally to this work.

\section{Acknowledgments}

This work was supported by the Technology Innovation Program (development of functional ingredients from organic rice as idle resource and its value-added applications) funded by the Ministry of Trade, Industry \& Energy (MOTIE, Korea).

\section{References}

[1] C. A. G. M. Weijers, M. C. R. Franssen, and G. M. Visser, "Glycosyltransferase-catalyzed synthesis of bioactive oligosaccharides," Biotechnology Advances, vol. 26, no. 5, pp. 436-456, 2008.

[2] C. Zhao, Y. Wu, X. Liu et al., "Functional properties, structural studies and chemo-enzymatic synthesis of oligosaccharides," Trends in Food Science \& Technology, vol. 66, pp. 135-145, 2017.

[3] X. Qiang, C. YongLie, and W. QianBing, "Health benefit application of functional oligosaccharides," Carbohydrate Polymers, vol. 77, no. 3, pp. 435-441, 2009.

[4] F. Fernández-Bañares, "Nutritional care of the patient with constipation," Best Practice \& Research Clinical Gastroenterology, vol. 20, no. 3, pp. 575-587, 2006.

[5] O. O. Ibrahim, "Functional oligo-saccharides: chemicals structure, manufacturing, health benefits, applications and regulations," Journal of Food Chemistry and Nanotechnology, vol. 4, no. 4, pp. 65-76, 2018.

[6] R. G. Crittenden and M. J. Playne, "Production, properties and applications of food-grade oligosaccharides," Trends in Food Science \& Technology, vol. 7, no. 11, pp. 353-361, 1996.

[7] Y. Okawa, S. Fukudo, and H. Sanada, "Specific foods can reduce symptoms of irritable bowel syndrome and functional constipation: a review," Biopsychosocial Medicine, vol. 13, no. 10, 2019.

[8] J. Muir, "An overview of fiber and fiber supplements for irritable bowel syndrome," Gastroenterology and Hepatology (N Y), vol. 15, no. 7, pp. 387-389, 2019.

[9] A. K. Goulas, D. A. Fisher, G. K. Grimble, A. S. Grandison, and R. A. Rastall, "Synthesis of isomaltooligosaccharides and oligodextrans by the combined use of dextransucrase and dextranase," Enzyme and Microbial Technology, vol. 35, no. 4, pp. 327-338, 2004.

[10] L. Wang, L. Hu, S. Yan et al., "Effects of different oligosaccharides at various dosages on the composition of gut microbiota and short-chain fatty acids in mice with constipation," Food \& Function, vol. 8, no. 5, pp. 1966-1978, 2017.
[11] E. Y. Kim, K. Jo, S. H. Ahn, S. S. Park, H. S. Son, and S. H. Han, "Effect of lactic acid bacteria powder on loperamide-induced constipation in rat," The Korean Journal of Food and Nutrition, vol. 28, no. 6, pp. 956-964, 2015.

[12] C. Rémésy and C. Remesy, "Stimulation of absorption of volatile fatty acids and minerals in the cecum of rats adapted to a very high fiber diet," The Journal of Nutrition, vol. 115, no. 1, pp. 53-60, 1985.

[13] M. Takaki, "Gut pacemaker cells: the interstitial cells of cajal (ICC)," Journal of Smooth Muscle Research, vol. 39, no. 5, pp. 137-161, 2003.

[14] M. R. Henrik, B. S. H. Lea, I. B. Martin et al., "Colonic transit time is related to bacterial metabolism and mucosal turnover in the gut," Nature Microbiology, vol. 1, no. 9, 2016.

[15] E. Hijova and A. Chmelarova, "Short chain fatty acids and colonic health," Bratislavske Lekarske Listy, vol. 108, no. 8, pp. 354-358, 2007.

[16] S. Yan, Y.-Z. Yue, M.-M. Sun, B.-S. Wu, and X.-P. Wang, "Suppressive effect of aurantii fructus immaturus and atractylodis macrocephalae rhizoma on glutamic acid-induced autophagy of interstitial cells of cajal," Journal of Integrative Medicine, vol. 18, no. 4, pp. 334-343, 2020.

[17] C. L. He, L. Burgart, L. Wang et al., "Decreased interstitial cell of cajal volume in patients with slow-transit constipation," Gastroenterology, vol. 118, no. 1, pp. 14-21, 2000.

[18] R. Walia, L. Mahajan, and R. Steffen, "Recent advances in chronic constipation," Current Opinion in Pediatrics, vol. 21, no. 5, pp. 661-666, 2009.

[19] I. J. D. McCallum, S. Ong, and M. Mercer-Jones, "Chronic constipation in adults," British Medical Journal, vol. 338, no. 831, pp. 763-766, 2009.

[20] F. W. Leung, "Etiologic factors of chronic constipation-review of the scientific evidence," Digestive Diseases and Sciences, vol. 52, no. 2, pp. 313-316, 2007.

[21] G. Fox, "Starch in brewingapplications," in Starch in Food, Structure, Function and Applications, S. Malin and N. Lars, Eds., pp. 633-659, Elsevier, Amsterdam, Netherlands, 2018.

[22] C. S. Lee, P. L. Tan, J. Y. Eor et al., "Prophylactic use of probiotic chocolate modulates intestinal physiological functions in constipated rats," Journal of the Science of Food and Agriculture, vol. 99, no. 6, pp. 3045-3056, 2019.

[23] N. A. P. Chukwuemeka, I. P. Adejoh, I. L. Osafanme, S. V. Duniya, and M. T. Boniface, "Laxative effects of aqueous extract of Sida acuta leaves in loperamide-induced constipation in wistar rats," Asian Journal of Research in Medical and Pharmaceutical Sciences, vol. 6, no. 1, pp. 1-7, 2019.

[24] S. H. Han, K. B. Hong, E. Y. Kim, S. H. Ahn, and H. J. Suh, "Effect of dual-type oligosaccharides on constipation in loperamide-treated rats," Nutrition Research and Practice, vol. 10 , no. 6 , pp. 583-589, 2016.

[25] S. H. Han, K. Park, E. Y. Kim et al., "Cactus (Opuntia humifusa) water extract ameliorates loperamide-induced constipation in rats," BMC Complementary Medicine and Therapies, vol. 17, no. 1, pp. 49-57, 2017.

[26] H. Guzman-Maldonado, O. Paredes-Lopez, and C. G. Biliaderis, "Amylolytic enzymes and products derived from starch: a review," Critical Reviews in Food Science and Nutrition, vol. 35, no. 5, pp. 373-403, 1995.

[27] D. Sasaki, K. Sasaki, N. Ikuta et al., "Low amounts of dietary fibre increase in vitro production of short-chain fatty acids without changing human colonic microbiota structure," Scientific Reports, vol. 8, no. 1, 2018.

[28] S. Xue, X. Chen, J. Lu, and L. Jin, "Protective effect of sulfated Achyranthes bidentata polysaccharides on streptozotocin- 
induced oxidative stress in rats," Carbohydrate Polymers, vol. 75, no. 3, pp. 415-419, 2009.

[29] A. W. Walker, J. Ince, S. H. Duncan et al., "Dominant and diet-responsive groups of bacteria within the human colonic microbiota," The ISME Journal, vol. 5, no. 2, pp. 220-230, 2011.

[30] D. Wu, J. Zhou, X. Wang et al., "Traditional Chinese formula, lubricating gut pill, stimulates cAMP-dependent $\mathrm{Cl}$ - secretion across rat distal colonic mucosa," Journal of Ethnopharmacology, vol. 134, no. 2, pp. 406-413, 2011.

[31] D. Cockburn, C. Wilkens, C. Ruzanski et al., "Analysis of surface binding sites (SBSs) in carbohydrate active enzymes with focus on glycoside hydrolase families 13 and 77-a minireview," Biologia, vol. 69, pp. 705-712, 2014.

[32] A. Shimotoyodome, S. Meguro, T. Hase, I. Tokimitsu, and T. Sakata, "Decreased colonic mucus in rats with loperamideinduced constipation," Comparative Biochemistry and Physiology Part A: Molecular \& Integrative Physiology, vol. 126, no. 2, pp. 203-211, 2000.

[33] M. Faderl, M. Noti, N. Corazza, and C. Mueller, "Keeping bugs in check: the mucus layer as a critical component in maintaining intestinal homeostasis," IUBMB Life, vol. 67, no. 4, pp. 275-285, 2015.

[34] A. Shimotoyodome, S. Meguro, T. Hase, I. Tokimitsu, and T. Sakata, "Sulfated polysaccharides, but not cellulose, increase colonic mucus in rats with loperamide-induced constipation," Digestive Diseases and Sciences, vol. 46, no. 7, pp. 1482-1489, 2001.

[35] A. J. Burns, T. M. Herbert, S. M. Ward, and K. M. Sanders, "Interstitial cells of cajal in the Guinea-pig gastrointestinal tract as revealed by c-kit immunohistochemistry," Cell and Tissue Research, vol. 290, no. 1, pp. 11-20, 1997.

[36] T. Wedel, J. Spiegler, S. Soellner et al., "Enteric nerves and interstitial cells of cajal are altered in patients with slow-transit constipation and megacolon," Gastroenterology, vol. 123, no. 5, pp. 1459-1467, 2002. 